\title{
Tuning the Structure and Hydrolysis Stability of Calcium Metal-Organic Frameworks through Integrating Carboxylic/ Phosphinic/ Phosphonic Groups in Building Blocks
}

Jing Sun, ${ }^{\dagger}$ Tao Huang,,$^{\dagger}$ Qi Yin,,$^{\dagger}$ Lan Li, ${ }^{\dagger}$ Tian-Fu Liu, ${ }^{\dagger}$ Xin-Song Huang ${ }^{* \dagger}$ and Rong Cao*ts

'State Key Laboratory of Structural Chemistry, Fujian Institute of Research on the Structure of Matter, Chinese Academy of Sciences, Fuzhou, 350002, P. R. China.

"School of Physical Science and Technology, ShanghaiTech University, Shanghai, 201210, P. R. China.

${ }^{\S}$ University of Chinese Academy of Sciences, Beijing, 100049, P. R. China 


\section{Material and Instruments}

All the chemicals were purchased from standard chemical suppliers and used without further purification. Calcium nitrate tetrahydrate $\left[\mathrm{Ca}\left(\mathrm{NO}_{3}\right)_{2} \cdot 4 \mathrm{H}_{2} \mathrm{O}\right.$, Aldrich 95\%] and BPDA $=\left[2,2^{\prime}\right]$ Bipyridinyl-5,5'-dicarboxylic acid were purchased from Tansoole. $\mathrm{COOH}-\mathrm{PO}_{3}=4$-Phosphono-benzoic acid was purchased from Shanghai Tensus Bio-tech CO., Ltd.

Single crystal X-ray diffraction data was collected on 1) an Oxford Diffraction SuperNova diffractometer equipped with $\mathrm{Cu}-\mathrm{K} \alpha$ and Mo-K $\alpha$ radiation $(\lambda=1.5418 \AA$, $0.7107 \AA), 2$ ) a BRUKER D8 VENTURE equipped with $\mathrm{Cu}-\mathrm{K} \alpha$ and Mo-K $\alpha$ radiation $(\lambda=1.5418 \AA, 0.7107 \AA$ ). PXRD was performed on Rikagu Miniflex 600 Benchtop X-ray diffraction instrument. TGA was performed on a Seiko S-II instrument, and the dried crystalline samples were heated at a rate of $5^{\circ} \mathrm{C} / \mathrm{min}$ up to $800^{\circ} \mathrm{C}$ and then cooled to room temperature under $\mathrm{N}_{2}$ atmosphere. The $\mathrm{N}_{2}$ gas isotherms of the samples were measured using ASAP 2020 and ASAP 2460 from Micromeritics Co. Ltd.

\section{Single-crystal X-ray Crystallography}

Single crystal X-ray diffraction data of PFC-36 was collected on Rigaku Oxford Diffraction SuperNova diffractometer equipped with Mo-K $\alpha$ radiation $(\lambda=0.71073 \AA)$ at 298 K. Single crystal X-ray diffraction data of PFC-38 was collected on Rigaku Oxford Diffraction SuperNova diffractometer equipped with $\mathrm{Cu}-\mathrm{K} \alpha$ radiation $(\lambda=1.5418 \AA)$ at $150 \mathrm{~K}$. Single crystal X-ray diffraction data of PFC-39 was collected on Bruker APEX-II CCD diffractometer equipped with $\mathrm{Cu}-\mathrm{K} \alpha$ radiation $(\lambda=1.5418 \AA)$ 
at 200.15 K. Single crystal X-ray diffraction data of PFC-40 was collected on Bruker APEX-II CCD diffractometer equipped with $\mathrm{Cu}-\mathrm{K} \alpha$ radiation $(\lambda=1.5418 \AA)$ at $100 \mathrm{~K}$. Single crystal X-ray diffraction data of PFC-41 was collected on Bruker APEX-II CCD diffractometer equipped with Mo-K $\alpha$ radiation $(\lambda=0.71073 \AA)$ at $100 \mathrm{~K}$. Final unit cell parameters were derived from the global refinements of reflections obtained from the integration of all frame data. The CrysAlisPro and APEX3 software package were used to determine unit cells and for data collection and data integration. The data were processed with SCALE3 ABSPACK scaling algorithm, SAINT and SADABS (TWINABS) and corrected for absorption using multi-scan techniques. The structure was solved with the ShelXT structure solution program using Intrinsic Phasing. Then using Olex $2^{1}$, the structure was refined with the ShelXL ${ }^{2}$ refinement package using Least Square minimization. Anisotropic thermal parameters were used for the non-hydrogen atoms. $\mathrm{H}$ atoms attached to carbon and hydroxyl oxygen atoms were positioned geometrically and constrained to ride on their parent atoms. PLATON SQUEEZE was employed to calculate the contribution to the diffraction from the solvent region and thereby produced a set of solvent-free diffraction intensities. More crystallographic details are given in Table S1. 
Table S1. Crystallographic data of PFC-36, PFC-38, PFC-39, PFC-40, and PFC-41

\begin{tabular}{|c|c|c|c|c|c|}
\hline Identification code & PFC-36 & PFC-38 & PFC-39 & PFC-40 & PFC-41 \\
\hline \multirow{2}{*}{ Empirical formula } & $\mathrm{C}_{12} \mathrm{H}_{6} \mathrm{CaN}_{2} \mathrm{O}_{5}$ & $\mathrm{C}_{116} \mathrm{H}_{100} \mathrm{Ca}_{5} \mathrm{~N}_{17} \mathrm{O}$ & $\mathrm{C}_{34} \mathrm{H}_{17} \mathrm{Ca}_{3} \mathrm{O}_{25}$ & $\mathrm{C}_{40} \mathrm{H}_{36} \mathrm{Ca}_{5} \mathrm{~N}_{2} \mathrm{O}_{33}$ & $\mathrm{C}_{28} \mathrm{H}_{20} \mathrm{Ca}_{4} \mathrm{O}_{24}$ \\
\hline & & 28 & $\mathrm{P}_{2}$ & $\mathrm{P}_{2}$ & $\mathrm{P}_{4}$ \\
\hline CCDC number & 2003104 & 2003105 & 2003106 & 2003107 & 2003108 \\
\hline Formula weight & 298.27 & 2380.52 & 1007.65 & 1335.05 & 1024.64 \\
\hline Temperature/K & 298.00 & 150.0 & 200.15 & 100.02 & 100.0 \\
\hline Crystal system & Monoclinic & Triclinic & Triclinic & Triclinic & Monoclinic \\
\hline Space group & $P 2{ }_{1} / \mathrm{c}$ & $P-1$ & $P-1$ & $P-1$ & $P \mathrm{n}$ \\
\hline $\mathrm{a} / \AA$ & $13.8511(8)$ & $17.0339(5)$ & $8.9670(3)$ & $13.6927(5)$ & $5.6747(4)$ \\
\hline $\mathrm{b} / \AA$ & $6.9167(5)$ & $17.1196(4)$ & $9.1832(3)$ & $16.2875(6)$ & $10.967(14)$ \\
\hline $\mathrm{c} / \AA$ & $11.8020(8)$ & $17.9227(6)$ & $17.6446(5)$ & $18.1025(7)$ & $38.723(3)$ \\
\hline$\alpha /{ }^{\circ}$ & 90 & $90.805(2)$ & $99.4230(10)$ & $71.497(2)$ & 90 \\
\hline$\beta /{ }^{\circ}$ & $95.629(7)$ & $116.455(3)$ & $93.1680(10)$ & $85.212(2)$ & 93.715 \\
\hline$\gamma /{ }^{\circ}$ & 90 & 117.979(3) & $104.125(10)$ & $87.664(2)$ & 90 \\
\hline Volume $/ \AA^{3}$ & $1125.23(13)$ & $3964.4(2)$ & $1383.11(8)$ & $3814.7(3)$ & $2404.8(4)$ \\
\hline Z & 4 & 1 & 1 & 2 & 2 \\
\hline$\rho_{\text {calcg }} / \mathrm{cm}^{3}$ & 1.761 & 1.003 & 1.210 & 1.164 & 1.415 \\
\hline$\mu / \mathrm{mm}^{-1}$ & 0.581 & 1.983 & 3.783 & 4.107 & 0.685 \\
\hline $\mathrm{F}(000)$ & 608 & 1239 & 511 & 1368 & 1040 \\
\hline Crystal size $/ \mathrm{mm}^{3}$ & $0.7 \times 0.08 \times 0.01$ & $0.3 \times 0.3 \times 0.01$ & $0.6 \times 0.4 \times 0.01$ & $0.8 \times 0.04 \times 0.01$ & $\begin{array}{l}1.2 \times 0.04 \times 0.0 \\
1\end{array}$ \\
\hline Radiation & $\begin{array}{l}\operatorname{MoK} \alpha(\lambda= \\
0.71073)\end{array}$ & $\begin{array}{l}\mathrm{CuK} \alpha \quad(\lambda= \\
1.54178)\end{array}$ & $\begin{array}{l}\operatorname{CuK} \alpha(\lambda= \\
1.54178)\end{array}$ & $\begin{array}{l}\operatorname{CuK} \alpha(\lambda= \\
1.54178)\end{array}$ & $\begin{array}{l}\operatorname{MoK} \alpha(\lambda= \\
0.71073)\end{array}$ \\
\hline $\begin{array}{l}2 \theta \text { range for data } \\
\text { collection }^{\circ}{ }^{\circ}\end{array}$ & $\begin{array}{l}5.904- \\
49.992\end{array}$ & $6.840-146.940$ & $\begin{array}{l}10.222- \\
140.138\end{array}$ & $5.162-107.764$ & $\begin{array}{l}4.878- \\
57.678\end{array}$ \\
\hline Reflections collected & 3457 & 28401 & 17100 & 72226 & 60412 \\
\hline $\begin{array}{l}\text { Independent } \\
\text { reflections }\end{array}$ & $\begin{array}{l}1913 \\
(\text { Rint }= \\
0.0672, \\
\text { Rsigma = } \\
0.0696)\end{array}$ & $\begin{array}{l}15441 \\
(\text { Rint }=0.0320, \\
\text { Rsigma }= \\
0.0407)\end{array}$ & $\begin{array}{l}4999 \\
(\text { Rint }= \\
0.0622, \\
\text { Rsigma = } \\
0.0639)\end{array}$ & $\begin{array}{l}9167 \\
(\operatorname{Rint}=0.2383, \\
\text { Rsigma }= \\
0.0950)\end{array}$ & $\begin{array}{l}12584 \text { (Rint } \\
=0.0988, \\
\text { Rsigma }= \\
0.0875)\end{array}$ \\
\hline $\begin{array}{l}\text { Data/restraints/param } \\
\text { eters }\end{array}$ & $1913 / 0 / 181$ & $15441 / 1 / 762$ & 4999/187/408 & $9167 / 360 / 752$ & $\begin{array}{l}12584 / 320 / 54 \\
2\end{array}$ \\
\hline Goodness-of-fit on $\mathrm{F}^{2}$ & 1.097 & 0.977 & 1.107 & 1.073 & 1.058 \\
\hline $\begin{array}{l}\text { Final } R \text { indexes }[I \geq 2 \sigma \\
\text { (I) }]\end{array}$ & $\begin{array}{l}\mathrm{R}_{1}=0.075 \\
\mathrm{wR}_{2}=0.259\end{array}$ & $\begin{array}{l}\mathrm{R}_{1}=0.0594, \\
{ }_{\mathrm{w}} \mathrm{R}_{2}=0.1718\end{array}$ & $\begin{array}{l}\mathrm{R}_{1}=0.1733 \\
{ }_{\mathrm{w}} \mathrm{R}_{2}=0.419\end{array}$ & $\begin{array}{l}\mathrm{R}_{1}=0.1063, \\
{ }_{\mathrm{w}} \mathrm{R}_{2}=0.2679\end{array}$ & $\begin{array}{l}\mathrm{R}_{1}=0.095 \\
\mathrm{wR}_{2}=0.257\end{array}$ \\
\hline $\begin{array}{l}\text { Final } R \text { indexes [all } \\
\text { data] }\end{array}$ & 0.1043 & 0.0711 & 0.1764 & 0.1131 & 0.1162 \\
\hline $\begin{array}{l}\text { Largest diff. peak / } \\
\text { hole / e } \AA^{-3}\end{array}$ & $0.85 /-0.92$ & $1.16 /-0.50$ & $2.16 /-1.32$ & $1.51 /-0.74$ & $1.61 /-3.43$ \\
\hline$\theta_{\max }[\mathrm{deg}]$ & 24.996 & 73.470 & 70.069 & 53.882 & 28.839 \\
\hline Completeness & $96 \%$ & $100 \%$ & $95 \%$ & $100 \%$ & $100 \%$ \\
\hline
\end{tabular}




\section{Crystal structures}

Table S2. Different coordination environment in the inorganic secondary building blocks of $\mathrm{CaO}_{6}, \mathrm{CaO}_{7}$ and $\mathrm{CaO}_{8}$

\begin{tabular}{|l|l|l|l|l|l|}
\hline Image & Name & label & Faces & Edges & Vertices \\
\hline Octahedron & $\mathrm{CaO}_{6}$ & 8 & 12 & 6 \\
\hline $\begin{array}{c}\text { Pentagonal } \\
\text { bipyramid }\end{array}$ & $\mathrm{CaO}_{7}$ & 10 & 15 & 7 \\
\hline
\end{tabular}




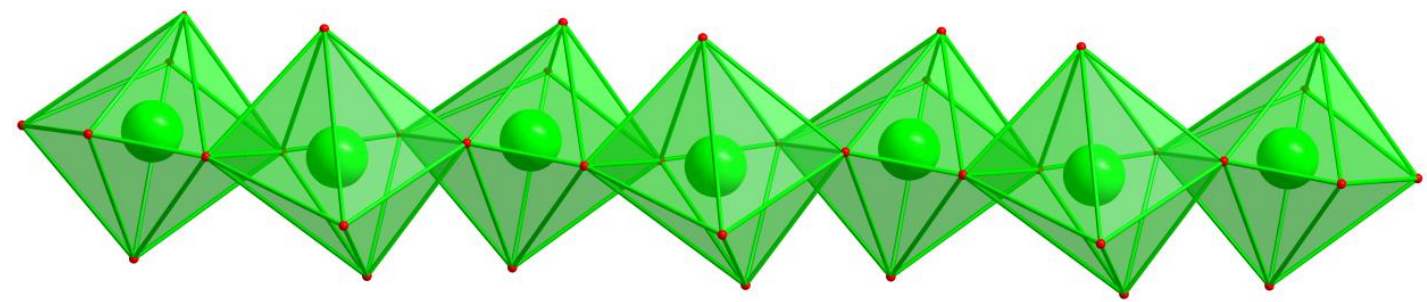

Figure $\mathrm{S} . \mathrm{CaO}_{7}$ chain-like building block in PFC-36 


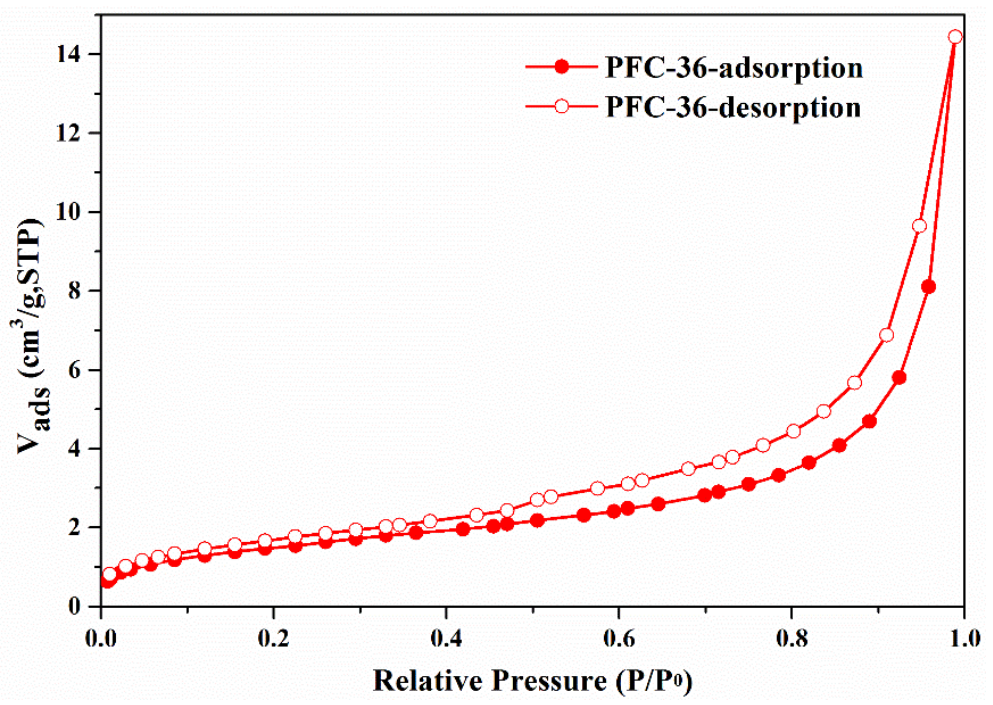

Figure S2. $\mathrm{N}_{2}$ adsorption at $77 \mathrm{~K}$ test of PFC-36 


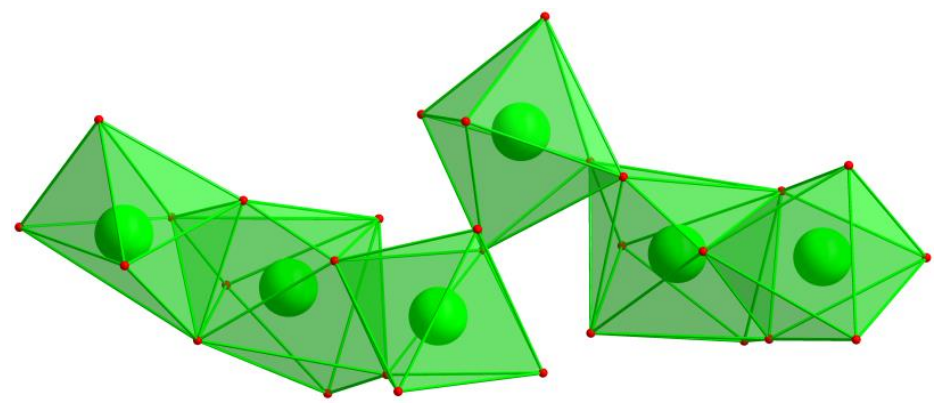

Figure S3. 6-nuclear $\mathrm{CaO}_{6}-\mathrm{CaO}_{8}-\mathrm{CaO}_{6}-\mathrm{CaO}_{6}-\mathrm{CaO}_{8}-\mathrm{CaO}_{6}$ rod-like building block in

PFC-37 

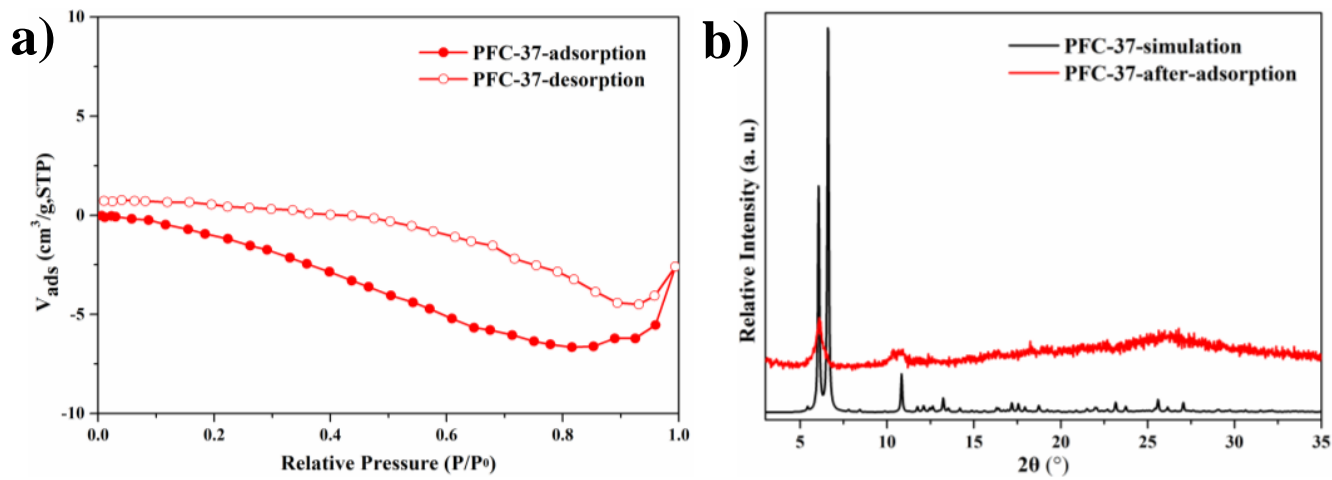

Figure S4. (a) $\mathrm{N}_{2}$ adsorption test at $77 \mathrm{~K}$ and (b) PXRD patterns of PFC-37 after-adsorption 


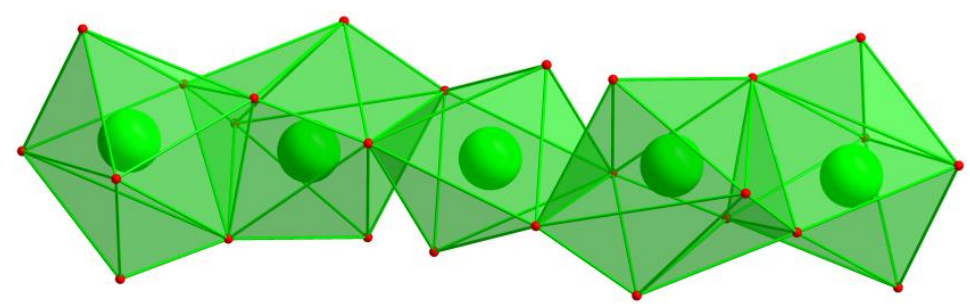

Figure S5. 5-nuclear $\mathrm{CaO}_{7}-\mathrm{CaO}_{8}-\mathrm{CaO}_{6}-\mathrm{CaO}_{8}-\mathrm{CaO}_{7}$ rod-like building block in PFC-38 


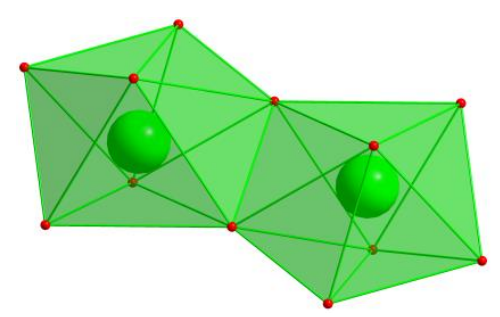

Figure S6. Binuclear $\mathrm{CaO}_{7}-\mathrm{CaO}_{7}$ bulk-like building block in PFC-39 


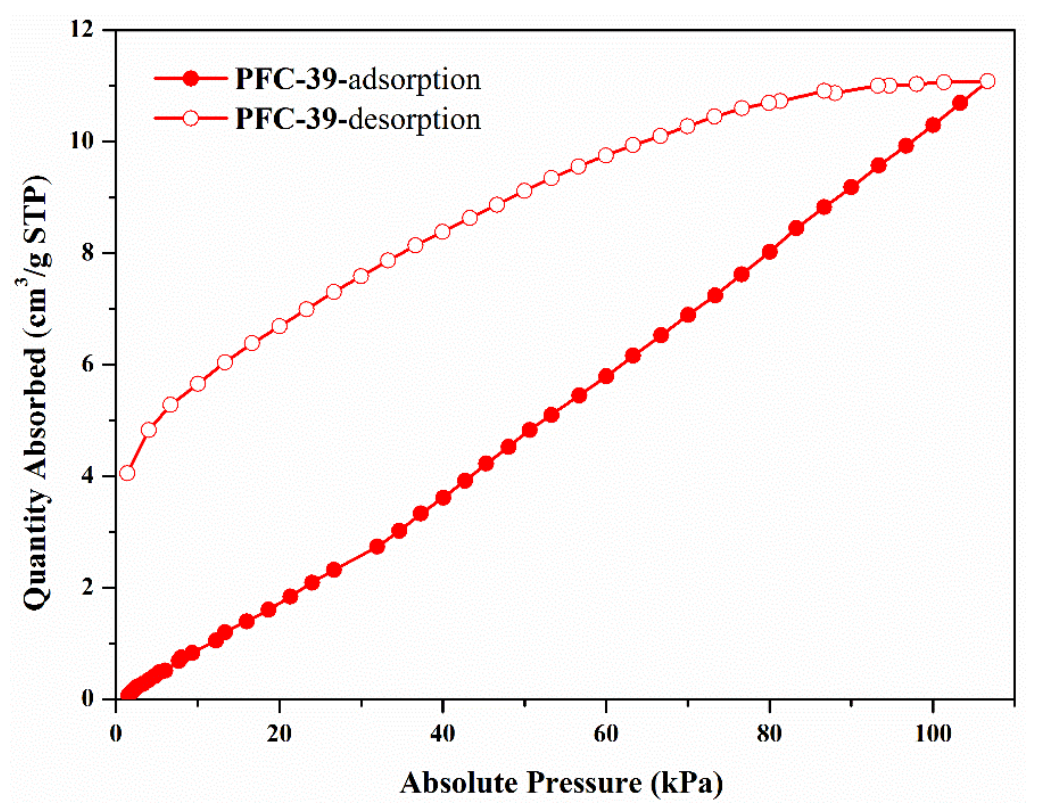

Figure S7. $\mathrm{CO}_{2}$ adsorption analysis at $298 \mathrm{~K}$ of PFC-39 
a)

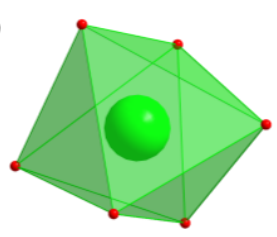

b)

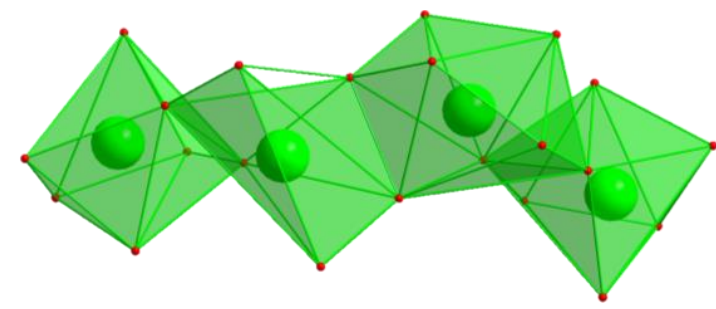

Figure S8. (a) The mononuclear $\mathrm{CaO}_{6}$ octahedron and (b) tetranuclear $\mathrm{CaO}_{7}-\mathrm{CaO}_{6}-\mathrm{CaO}_{8}-\mathrm{CaO}_{7}$ rod-like building blocks in PFC-40 


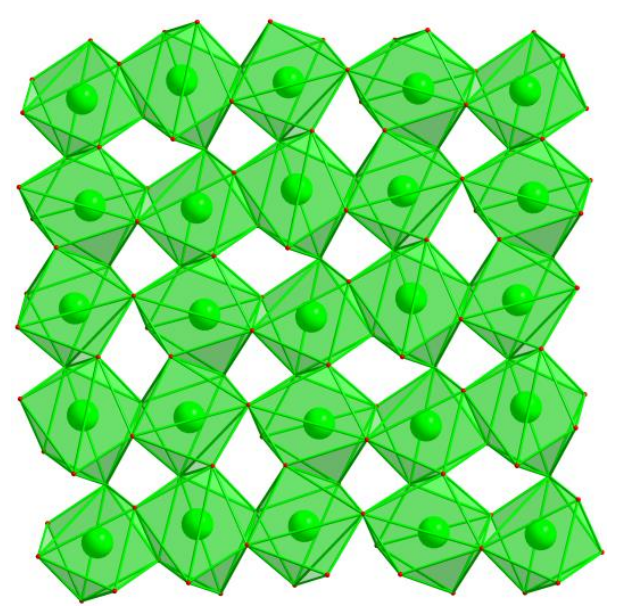

Figure S9. The $-\mathrm{CaO}_{7}-\mathrm{CaO}_{8}-\mathrm{CaO}_{7}-\mathrm{CaO}_{8}-$ chain formed a planar $\mathrm{Ca}-\mathrm{SBU}$ with a dislocation stacking method in PFC-41 


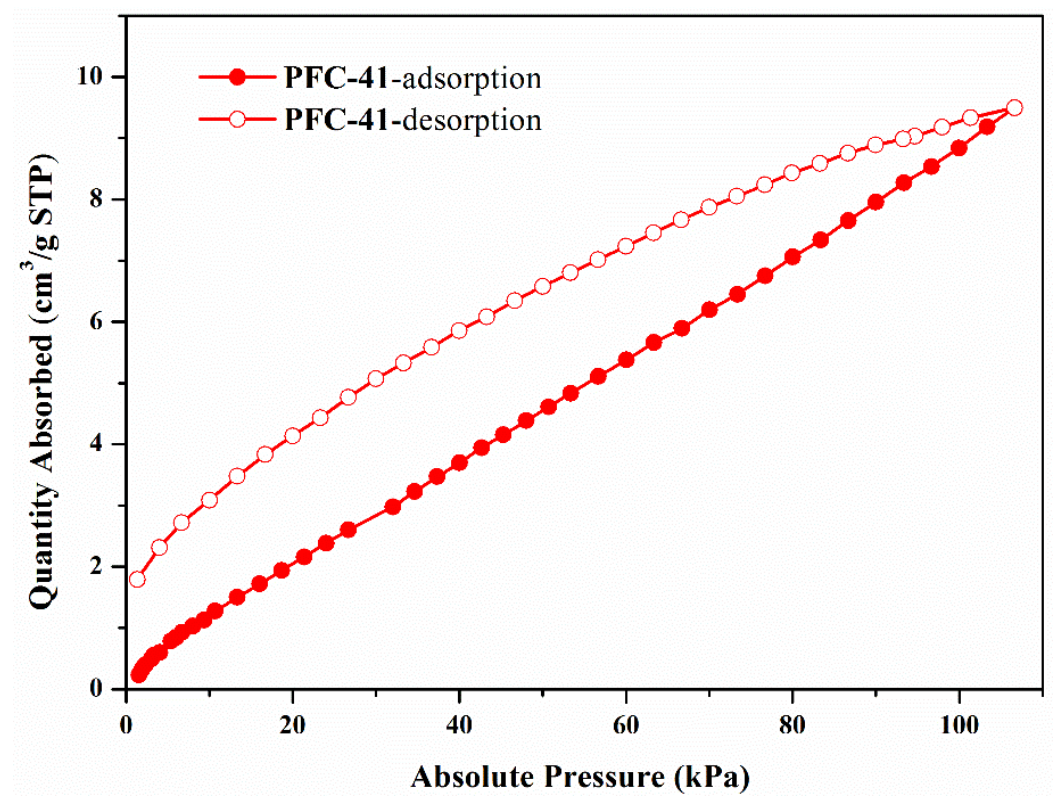

Figure $\mathrm{S} 10 . \mathrm{CO}_{2}$ adsorption analysis at $298 \mathrm{~K}$ of PFC-41 


\section{Drugs loading determination}

For cisplatin loading, $30 \mathrm{mg}$ activated PFC-41 was dispersed in $5 \mathrm{~mL}$ cisplatin aqueous solution $(10 \mathrm{mg} / \mathrm{ml})$ and the solution was put on the shaker for two days. The supernatant was then taken out for UV testing. The concentration of cisplatin aqueous solution was monitored by the absorbance at $\lambda=310 \mathrm{~nm}$ (Figure S11).

For 5-fluorouracil loading, the same procedures as the above except that cisplatin aqueous solution was replaced by 5 -fluorouracil $(5-\mathrm{Fu})$, and the concentration of 5 -fluorouracil aqueous solution was monitored by the absorbance at $\lambda=265 \mathrm{~nm}$ (Figure S12).

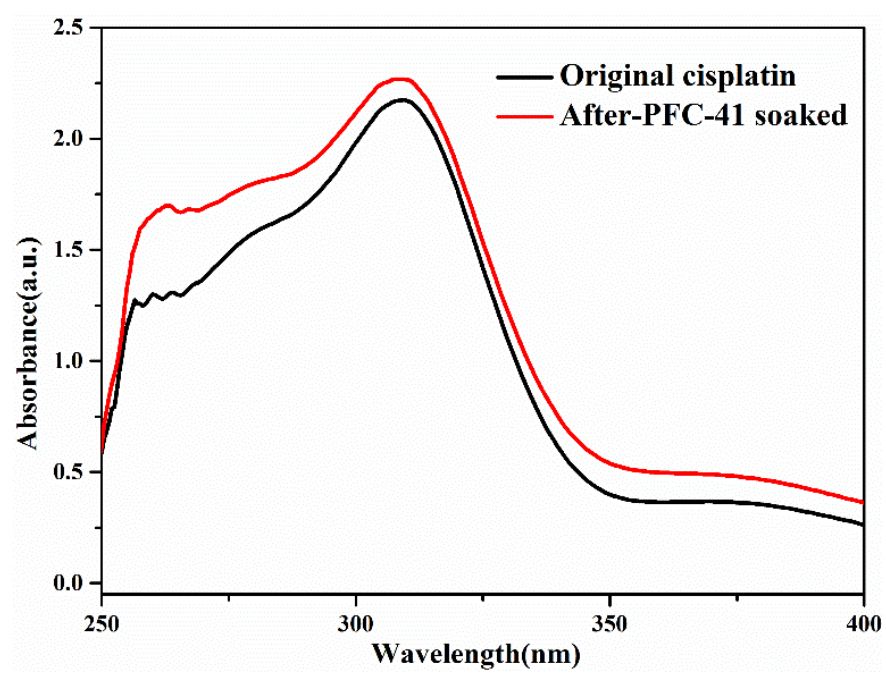

Figure S11. UV-Vis spectra of the supernatant of cisplatin solutions before (black) and after (red) addition of PFC-41 for 48 hours. 


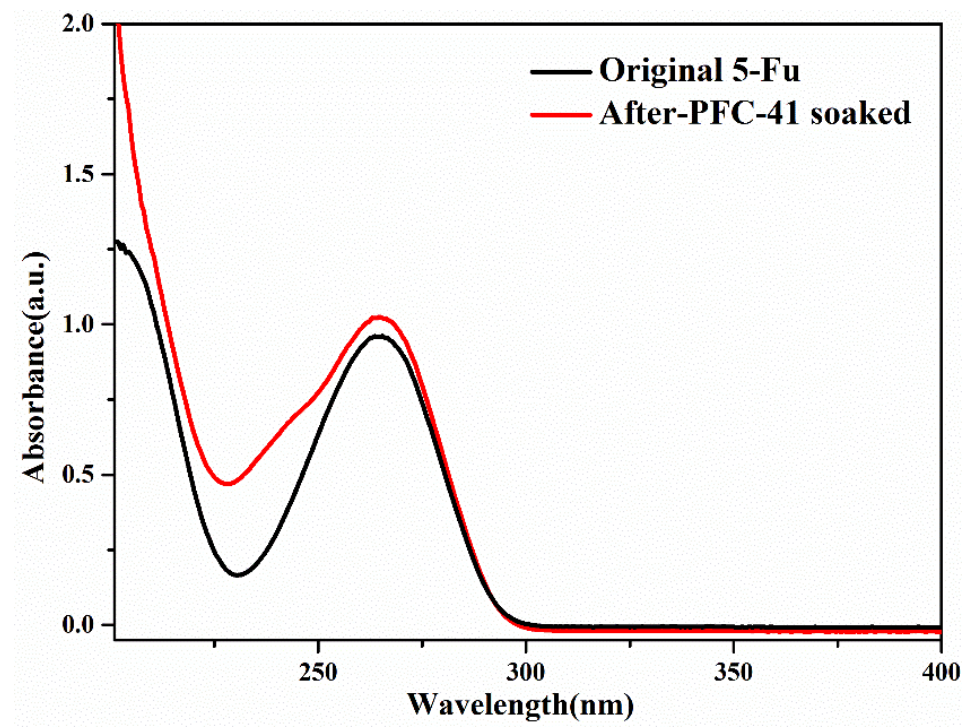

Figure S12. UV-Vis spectra of the supernatant of 5-Fu solutions before (black) and after (red) addition of PFC-41 for 48 hours. 
Urea loading determination

$30 \mathrm{mg}$ of PFC-41, previously dehydrated $\left(120^{\circ} \mathrm{C}\right.$ for $\mathrm{PFC}-41$ for $\left.12 \mathrm{~h}\right)$, was suspended into $5 \mathrm{~mL}$ of an aqueous solution of the urea $(500 \mathrm{mg} / \mathrm{ml})$ under stirring. After 53 hours, the urea-containing solids were recovered by centrifugation at 12000 rpm for $3 \mathrm{~min}$ and dried in air. The amount of urea into the materials was quantified by TGA, and the loading content is $57.2 \mathrm{wt} \%$ (Figure S13). The integrity of the loaded samples was examined by PXRD, and no damage to the crystallinity of PFC-41 was found (Figure S14).

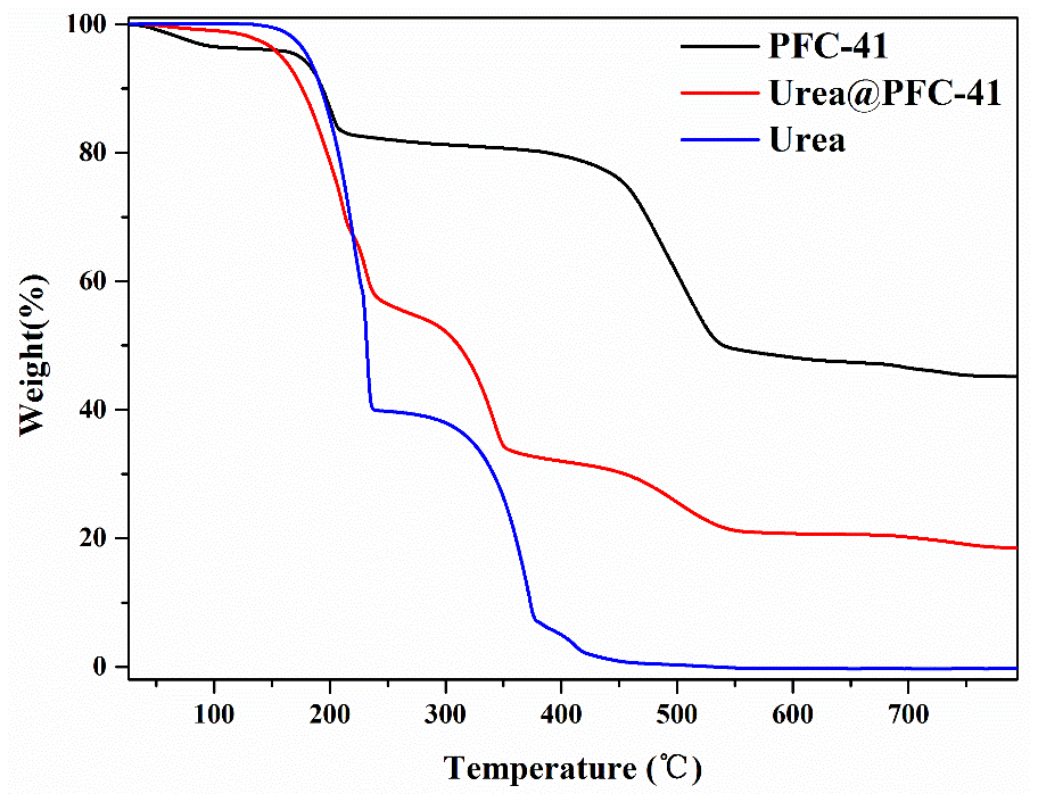

Figure S13. Thermogravimetric analysis of urea, PFC-41, and urea@PFC-41 


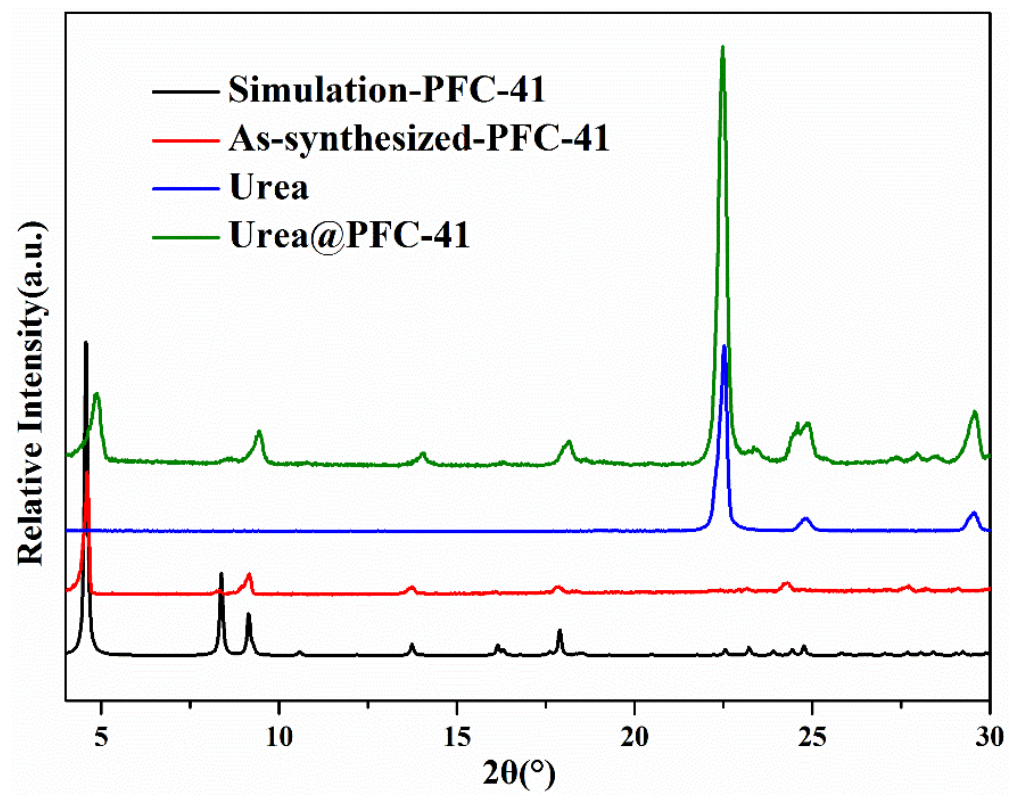

Figure S14. PXRD patterns of PFC-41, urea and urea@PFC-41 


\section{Thermogravimetric analysis}

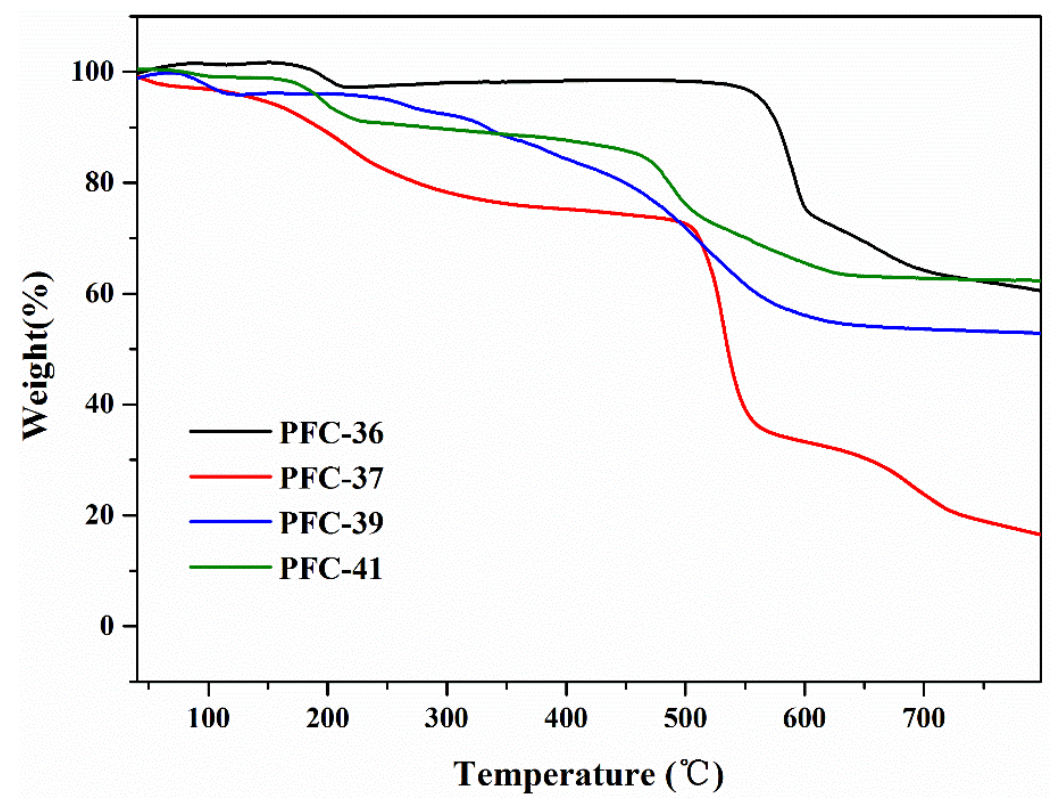

Figure S15. Thermogravimetric analysis of PFC-36, PFC-37, PFC-39 and PFC-41 


\section{IR spectra}

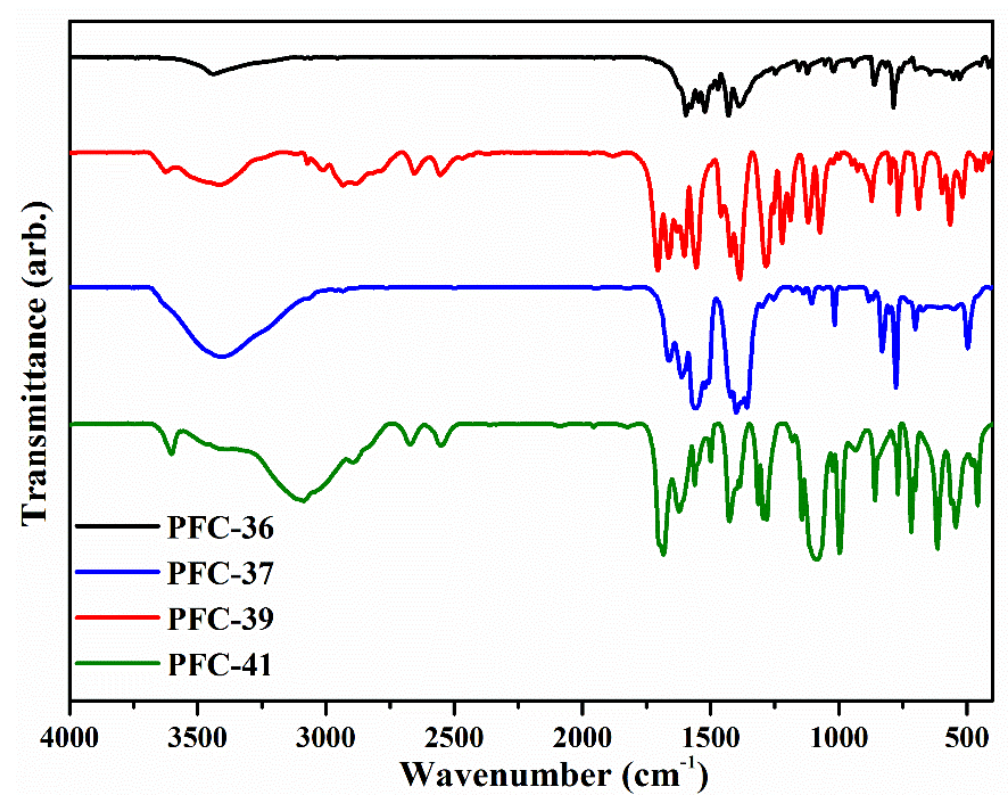

Figure S16. IR spectra of PFC-36, PFC-37, PFC-39 and PFC-41 


\section{References}

(1) Dolomanov, O. V.; Bourhis, L. J.; Gildea, R. J.; Howard, J. A. K.; Puschmann, H., OLEX2: a complete structure solution, refinement and analysis program. J. Appl. Crystallogr. 2009, 42, 339-341.

(2) Sheldrick, G. M., A short history of SHELX. Acta Crystallogr., Sect. A: Found. $A d v$. 2008, 64, 112-122. 